\title{
The Evaluation Analysis of Computer Network Information Security based on FAHP
}

\author{
Qian $\mathrm{Wu}$ \\ Weifang University of Science and Technology, Weifang, Shandong \\ 149360125@qq.com
}

\begin{abstract}
Computer network information security has attracted much attention of the society. The importance of computer network information is widely accepted. According to the determined selection criterion of computer network information security evaluation indexes to choose 5 first-level evaluation indexes and 19 second-level evaluation indexes to construct the evaluation system of computer network information security, using the analytic hierarchy process (AHP) to determine the weight of each evaluation index, the evaluation results of computer network information security are divided into 5 levels, that is, very safe, safe, general, dangerous, very dangerous. Establishing the fuzzy comprehensive evaluation model, through the expert evaluation method to carry on the level evaluation, the computer network information security evaluation of Weifang University of Science and Technology is taken as an example to carry on the simulation calculation. The fuzzy comprehensive evaluation model of the computer network information security is professional, scientific, and reasonable, which could provide theoretical basis for the development of computer network.
\end{abstract}

Keywords: network information; weight; AHP; computer; level evaluation

\section{Introduction}

With the progress of science and technology and the unceasing enhancement of network information degree of the whole society, the importance of computer network information has been widely accepted. Computer network information system has been extensive use in the business activities and government organizations. Computer network has become an indispensable tool in the social and economic development and people's daily lives. While computer information network technology brings convenience to people, it also brings threats. The openness of computer network information system determines that its development and application will suffer from viruses, Trojan, hacker, physical faults, and man-made destruction. The problems of computer network information security are increasingly prominent and gradually begin to be regarded.

Foreign researches on computer network information security evaluation can be traced back to twenty years ago. The IT developed countries such as the United States, Canada and so on have already established national certificate authorities and safety evaluation certificate systems in the 1970s, which are responsible for studying and developing the related evaluation criteria, evaluation authentication methods and evaluation technology, and carrying on the network security evaluation and certificate based on evaluation criteria. The evaluation work of computer network information security in our country is gradually developing in the constant understanding process of computer network information security. The computer network information security problems are put forward in the 1980s. But because of the insufficient understanding of risks, the absolute safety measures are usually taken. Until the 1990s, computer network information system was carried on the security level protection in our country. A series of related technical 
criteria and management norms were gradually put forward. The current main work of computer network information security focuses on the establishment of business system organization structure. The corresponding standard system and technology system are still in research stage.

Computer network information security evaluation is the foundation of network information security management, which is of great significance to computer network information security and management. Computer network information security evaluation makes clear to the current situation of computer network information security. After the network information security evaluation, the current situation of their own network, various application system and management system specification could be accurately understood so as to determine the safety needs of the organization. Computer network information security evaluation could determine the main risks of network information security so as to make each computer user choose the avoiding, reducing, or accepting risk management measures. Computer network information security evaluation can analyze all kinds of security risk factors; guide the construction of technology system and management system of computer network information security, and make the security strategy and security solutions of its network and system.

\section{The Evaluation Index System of Computer Network Information Security}

Computer network information security evaluation is a tedious work. Whether the evaluation indexes of computer network information security are reasonable and scientific or not concerns the evaluation function. The selected indexes are too many, and in fact they would be repetitive indexes, which could be interference. The selected indexes are too few, they would be lack of representativeness, which could be one-sidedness. Computer network information security evaluation needs to fully consider the influencing factors of computer network information security. Not only the fixed indexes should be considered, but also the variable indexes should be considered. In the selecting process of evaluation indexes, certain principles should be followed. The detailed principles are shown as follows:

\section{(1)Scientificity and comprehensiveness}

Establishing the evaluation index system of computer network information security must be based on scientific guidance, reflect the real nature of the object and the main factors influencing computer network information security.

Only insisting on the scientific principle, the obtained information could be reliable and objective, the evaluation results are valid. The computer network information security evaluation is a comprehensive evaluation of multi-indexes. In order to guarantee this, the selected indexes should be representative. When selecting, the various aspects of computer network information security evaluation should be considered.

\section{(2)Accuracy and fuzziness}

In the selection process of computer network information security, most selected indexes should be found the accurate data. According to the data, computer network information security is evaluated. Some indexes have no accurate data, but the evaluation direction or trend should be definite. Therefore, the accuracy and fuzziness should be combined.

(3)The combination of operability and systematicness

The established evaluation index system should be able to facilitate the data collection, reflect the comparability, make the evaluation procedures and work simplify as far as 
possible avoiding paying attention to all sides of a matter. Only having the feasibility, the evaluation implementation scheme could be easily accepted by tested unit.

(4)Comparability and stability

For comparison, the evaluation factors should be quantified. Computer network information security includes not only network security technology but network security management as well. The evaluation is much complex, which had better be quantified. At the same time, when establishing the evaluation indexes, the selected indexes should have the characteristics of regular change. The indexes that affected by accidental factors could not be selected.

On the basis of the four selection criteria of computer network information security indexes above, looking through a large number of reference, the evaluation index system of computer network information security is determined as 5 first-level evaluation indexes and 19 second-level evaluation indexes. The detailed indexes are shown in table 1.

Table 1. The Evaluation System of Computer Network Information Security

\begin{tabular}{|c|c|c|}
\hline \multirow{6}{*}{$\begin{array}{l}\text { Computer network } \\
\text { information security } \\
\text { evaluation } \mathrm{P}\end{array}$} & $\begin{array}{l}\text { first-level evaluation } \\
\text { indexes }\end{array}$ & second-level evaluation indexes \\
\hline & $\begin{array}{l}\text { network } \\
\text { communications } \\
\text { security A }\end{array}$ & $\begin{array}{l}\text { communications line security } \\
\mathrm{A}_{1} ; \text { communications control device } \\
\text { security } \mathrm{A}_{2} ; \text { encryption measures } \mathrm{A} \\
{ }_{3} ; \text { access control } \mathrm{A}_{4} ;\end{array}$ \\
\hline & $\begin{array}{l}\text { Information security } \\
\text { technology B }\end{array}$ & $\begin{array}{l}\text { security auditing function } \mathrm{B}_{1} ; \text { system } \\
\text { operation } \log \mathrm{B}_{2} ; \text { server backup } \mathrm{B}_{3} ; \text { anti- } \\
\text { hacker attack technology } \mathrm{B}_{4} ; \text {;antivirus } \\
\text { technology } \mathrm{B}_{5}\end{array}$ \\
\hline & System security C & $\begin{array}{l}\text { database security } \mathrm{C}_{1} \text {; application software } \\
\text { security } \mathrm{C}_{2} \text {; User authentication } \mathrm{C}_{3} \text {; user } \\
\text { information backup } \mathrm{C}_{4} \text {; }\end{array}$ \\
\hline & $\begin{array}{c}\text { Information } \\
\text { physical security D }\end{array}$ & $\begin{array}{l}\text { equipment security D1;environment } \\
\text { security D2:media security D3 }\end{array}$ \\
\hline & $\begin{array}{l}\text { Information security } \\
\text { system E }\end{array}$ & $\begin{array}{l}\text { rules and regulations } E_{1} ; \text { organization } \\
E_{2} ; \text { emergency plan } E_{3}\end{array}$ \\
\hline
\end{tabular}

\section{The Evaluation Model of Computer Network Information Security}

\subsection{Use the Analytic Hierarchy Process (AHP) to calculate the evaluation index weight of computer network information security}

Analytic hierarchy process (AHP) is a hierarchical and systematic method combining qualitative with quantitative. It could transform semi-qualitative and semi-quantitative problems into quantitative problems, through level-by-level comparison of a variety of related factors to provide data basis for analysis and forecast, deciding, and controlling things. The specific process is shown as follows:

1. According to the evaluation system of computer network information security, the hierarchical structure Figure of computer network information security evaluation is established. The specific is shown in figure 1. 


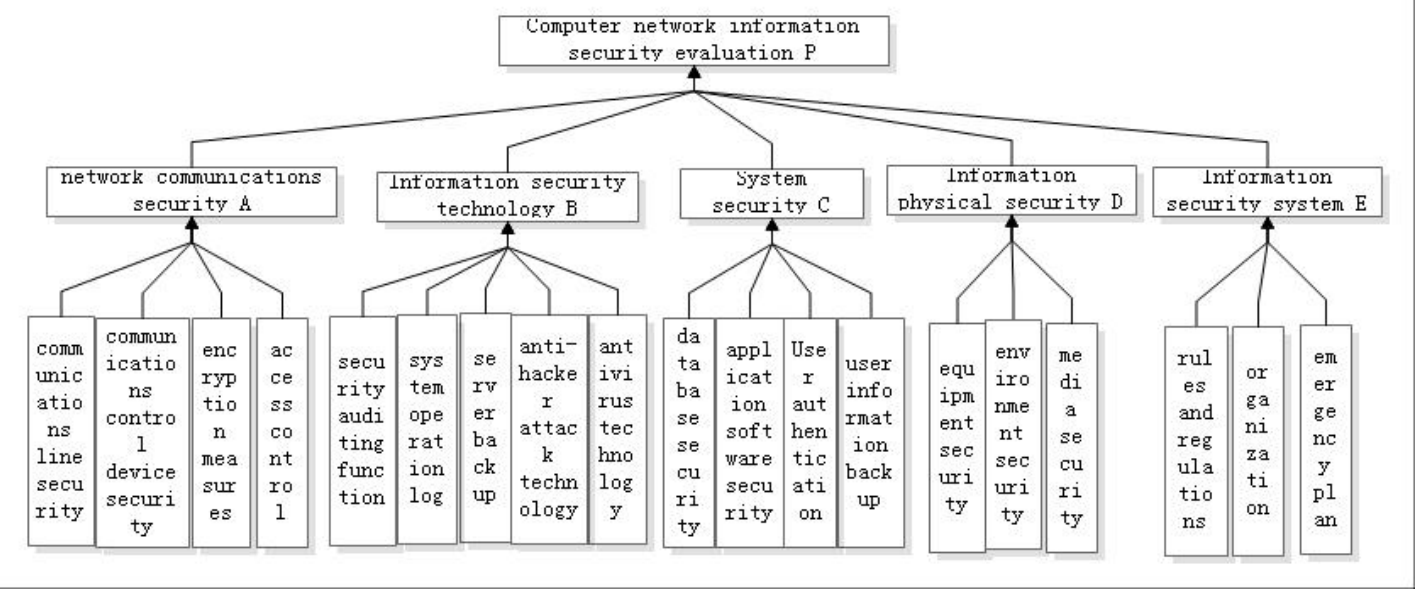

Figure 1. The Hierarchical Structure Figure of Computer Network Information Security Evaluation

2. Construct the comparison matrix of computer network information security evaluation.

Construct the comparison matrix by the experts comparing the influence each index at the same level gives to the index at the upper level. In the comparing process, 1-9 scale value is used. The details are shown in table 2.

Table 2. 1-9 Scale Value

\begin{tabular}{cccccccccc}
\hline scale $\mathrm{a}_{\mathrm{ij}}$ & 1 & 2 & 3 & 4 & 5 & 6 & 7 & 8 & 9 \\
\hline $\begin{array}{c}\text { The comparison } \\
\text { between index } \mathrm{i} \\
\text { and index } \mathrm{j}\end{array}$ & $\begin{array}{c}\text { The } \\
\text { same }\end{array}$ & $\begin{array}{l}\text { slightly } \\
\text { strong }\end{array}$ & strong & $\begin{array}{c}\text { Obviously } \\
\text { strong }\end{array}$ & $\begin{array}{c}\text { Absolute } \\
\text { strong }\end{array}$ \\
\hline
\end{tabular}

3. The determination of the evaluation index weight vector of computer network information security

Geometric mean method is used to calculate the weight vector of the indexes at all levels. There are three steps as follows:

(1)Calculate the element product of each line in comparison matrix, and we could get product vector $\alpha$;

(2)Carry on the square root $n$ times to vector $\alpha$, and we could get vector $\beta$.

(3)Carry on the normalization processing to vector $\beta$, and we could get index weight vector $\gamma$,

4. Carry on the consistency check to the calculated weight results.

There are many human factors in the determination of comparison matrix. So a consistency check is needed. The check process is divided into three steps:

(1)Calculate the consistency index: $C I=\frac{\lambda_{\max }-n}{n-1}$, and $\lambda_{\max }=\frac{1}{n} \sum_{i=1}^{n} \frac{\sum_{j=1}^{n} a_{i j} r_{j}}{r_{i}}$ is the maximum eigenvalue.

(2)According to $n$ to determine the random consistency index $R I$, the specific values are shown in table 3 . 
Table 3. The Random Consistency Index

\begin{tabular}{ccccccccccccc}
\hline $\mathrm{n}$ & 1 & 2 & 3 & 4 & 5 & 6 & 7 & 8 & 9 & 10 & 11 & 12 \\
\hline $\mathrm{RI}$ & 0 & 0 & 0.58 & 0.90 & 1.12 & 1.24 & 1.32 & 1.41 & 1.45 & 1.49 & 1.51 & 1.54 \\
\hline
\end{tabular}

(3)Calculate the consistency ratio $C R=\frac{C I}{R I}$, when $C R<0.10$, the comparison matrix could pass the check.

The calculated weight data of computer network information security is shown in table 4 to table 9 .

Table 4. The Comparison Matrix and the Check Results of the Target Layer

\begin{tabular}{|c|c|c|c|c|c|c|c|c|}
\hline target layer & \multicolumn{6}{|c|}{ computer network information security evaluation $\mathrm{P}$} & \multirow[b]{2}{*}{$\begin{array}{c}\text { The } \\
\text { largest } \\
\text { eigenvalue }\end{array}$} & \multirow[b]{2}{*}{$\begin{array}{c}\text { The } \\
\text { consistency } \\
\text { ratio }\end{array}$} \\
\hline $\begin{array}{l}\text { first-level } \\
\text { evaluation } \\
\text { indexes }\end{array}$ & $\begin{array}{c}\text { network } \\
\text { communications } \\
\text { security A }\end{array}$ & \begin{tabular}{|} 
Information \\
security \\
technology \\
B
\end{tabular} & \begin{tabular}{|c|} 
System \\
security \\
C
\end{tabular} & $\begin{array}{l}\text { Information } \\
\text { physical } \\
\text { security D }\end{array}$ & $\begin{array}{c}\text { Information } \\
\text { security } \\
\text { system E }\end{array}$ & weight & & \\
\hline \begin{tabular}{|c|} 
network \\
communications \\
security A
\end{tabular} & 1 & 3 & $1 / 4$ & 5 & 3 & 0.2162 & \multirow{5}{*}{5.1449} & \multirow{5}{*}{0.0324} \\
\hline $\begin{array}{c}\text { Information } \\
\text { security } \\
\text { technology B } \\
\end{array}$ & $1 / 3$ & 1 & $1 / 6$ & 2 & $1 / 2$ & 0.0747 & & \\
\hline $\begin{array}{c}\text { System security } \\
\text { C }\end{array}$ & 4 & 6 & 1 & 9 & 6 & 0.5586 & & \\
\hline $\begin{array}{c}\text { Information } \\
\text { physical } \\
\text { security D }\end{array}$ & $1 / 5$ & $1 / 2$ & $1 / 9$ & 1 & $1 / 3$ & 0.0435 & & \\
\hline $\begin{array}{c}\text { Information } \\
\text { security system } \\
\text { E }\end{array}$ & $1 / 3$ & 2 & $1 / 6$ & 3 & 1 & 0.1070 & & \\
\hline
\end{tabular}




\section{Table 5. The Comparison Matrix and the Check Results of First-level} Evaluation Index, Network Communications Security A

\begin{tabular}{|c|c|c|c|c|c|c|c|}
\hline $\begin{array}{l}\text { first-level } \\
\text { evaluation }\end{array}$ & \multicolumn{5}{|c|}{ network communications security A } & \multirow{2}{*}{$\begin{array}{c}\text { The } \\
\text { largest } \\
\text { eigenvalue }\end{array}$} & \multirow{2}{*}{$\begin{array}{c}\text { The } \\
\text { consistency } \\
\text { ratio }\end{array}$} \\
\hline $\begin{array}{c}\text { second-level } \\
\text { evaluation } \\
\text { indexes }\end{array}$ & $\begin{array}{l}\text { communications } \\
\text { line security } \mathrm{A} 1\end{array}$ & $\begin{array}{c}\text { communications } \\
\text { control device } \\
\text { security A } 2\end{array}$ & $\begin{array}{c}\text { encryption } \\
\text { measures } \\
\text { A } 3\end{array}$ & $\begin{array}{c}\text { access } \\
\text { control } \\
\text { A } 4\end{array}$ & weight & & \\
\hline $\begin{array}{l}\text { communications } \\
\text { line security A1 }\end{array}$ & 1 & $1 / 6$ & 3 & 2 & 0.1595 & \multirow{4}{*}{4.1152} & \multirow{4}{*}{0.0431} \\
\hline $\begin{array}{c}\text { communications } \\
\text { control device } \\
\text { security A } 2\end{array}$ & 6 & 1 & 7 & 8 & 0.6830 & & \\
\hline $\begin{array}{c}\text { encryption } \\
\text { measures A } 3\end{array}$ & $1 / 3$ & $1 / 7$ & 1 & $1 / 2$ & 0.0627 & & \\
\hline $\begin{array}{c}\text { access control A } \\
4\end{array}$ & $1 / 2$ & $1 / 8$ & 2 & 1 & 0.0949 & & \\
\hline
\end{tabular}

Table 6. The Comparison Matrix and the Check Results of First-level Evaluation Index, Information Security Technology B

\begin{tabular}{|c|c|c|c|c|c|c|c|c|}
\hline first-level & & inform & ation sec & curity techn & ology B & & & \\
\hline second-level & $\begin{array}{l}\text { security } \\
\text { auditing }\end{array}$ & $\begin{array}{c}\text { system } \\
\text { operatior }\end{array}$ & $\begin{array}{l}\text { server } \\
\text { backup }\end{array}$ & $\begin{array}{c}\text { anti- } \\
\text { hacker } \\
\text { attack }\end{array}$ & $\begin{array}{l}\text { antivirus } \\
\text { technology }\end{array}$ & yeight & $\begin{array}{c}\text { The } \\
\text { largest } \\
\text { eigenvalue }\end{array}$ & $\begin{array}{c}\text { The } \\
\text { consistency } \\
\text { ratio }\end{array}$ \\
\hline evaluation indexes & $\begin{array}{c}\text { function } \\
\text { B1 }\end{array}$ & $\log B 2$ & B3 & $\begin{array}{c}\text { technology } \\
\text { B4 }\end{array}$ & B5 & & & \\
\hline $\begin{array}{l}\text { security auditing } \\
\text { function } B 1\end{array}$ & 1 & 5 & 4 & $1 / 4$ & 3 & 0.2217 & & \\
\hline $\begin{array}{c}\text { system operation } \\
\log \mathrm{B} 2\end{array}$ & $1 / 5$ & 1 & $1 / 2$ & $1 / 8$ & $1 / 5$ & 0.0389 & & \\
\hline server backup B3 & $1 / 4$ & 2 & 1 & $1 / 7$ & $1 / 2$ & 0.0662 & 5.2623 & 0.0585 \\
\hline $\begin{array}{c}\text { anti-hacker attack } \\
\text { technology B4 }\end{array}$ & 4 & 8 & 7 & 1 & 7 & 0.5619 & & \\
\hline $\begin{array}{c}\text { antivirus } \\
\text { technology B5 }\end{array}$ & $1 / 3$ & 5 & 2 & $1 / 7$ & 1 & 0.1112 & & \\
\hline
\end{tabular}




\section{Table 7. The Comparison Matrix and the Check Results of First-level Evaluation Index, System Security C}

\begin{tabular}{|c|c|c|c|c|c|c|c|}
\hline $\begin{array}{l}\text { first-level } \\
\text { evaluation }\end{array}$ & \multicolumn{5}{|c|}{ system security $\mathrm{C}$} & \multirow{2}{*}{$\begin{array}{c}\text { The } \\
\text { largest } \\
\text { eigenvalue }\end{array}$} & \multirow{2}{*}{$\begin{array}{c}\text { The } \\
\text { consistency } \\
\text { ratio }\end{array}$} \\
\hline $\begin{array}{l}\text { second-level } \\
\text { evaluation } \\
\text { indexes }\end{array}$ & $\begin{array}{c}\text { database } \\
\text { security } \\
\text { C } 1\end{array}$ & $\begin{array}{c}\text { application } \\
\text { software } \\
\text { security C } \\
2\end{array}$ & $\begin{array}{c}\text { user } \\
\text { authentication } \\
\text { C } 3\end{array}$ & $\begin{array}{c}\text { user } \\
\text { information } \\
\text { backup C4 }\end{array}$ & weight & & \\
\hline $\begin{array}{c}\text { database } \\
\text { security C } 1\end{array}$ & 1 & $1 / 5$ & $1 / 3$ & $1 / 4$ & 0.0736 & \multirow{4}{*}{4.0514} & \multirow{4}{*}{0.0192} \\
\hline $\begin{array}{l}\text { application } \\
\text { software } \\
\text { security C } 2\end{array}$ & 5 & 1 & 3 & 2 & 0.4709 & & \\
\hline $\begin{array}{c}\text { User } \\
\text { authentication } \\
\text { C 3 }\end{array}$ & 3 & $1 / 3$ & 1 & $1 / 2$ & 0.1715 & & \\
\hline $\begin{array}{c}\text { user } \\
\text { information } \\
\text { backup C4 }\end{array}$ & 4 & $1 / 2$ & 2 & 1 & 0.2840 & & \\
\hline
\end{tabular}

Table 8. The Comparison Matrix and the Check Results of First-level Evaluation Index, Information Physical Security D

\begin{tabular}{|c|c|c|c|c|c|c|}
\hline first-level evaluation indexes & \multicolumn{4}{|c|}{ information physical security D } & \multirow[b]{2}{*}{$\begin{array}{c}\text { The } \\
\text { largest } \\
\text { eigenvalue }\end{array}$} & \multirow[b]{2}{*}{$\begin{array}{c}\text { The } \\
\text { consistency } \\
\text { ratio }\end{array}$} \\
\hline second-level evaluation indexes & $\begin{array}{l}\text { equipment } \\
\text { security } \\
\text { D1 }\end{array}$ & $\begin{array}{l}\text { environment } \\
\text { security D2 }\end{array}$ & $\begin{array}{l}\text { media } \\
\text { security } \\
\text { D3 }\end{array}$ & weight & & \\
\hline equipment security D1 & 1 & 3 & $1 / 3$ & 0.2499 & \multirow{3}{*}{3.0183} & \multirow{3}{*}{0.0176} \\
\hline environment security D2 & $1 / 3$ & 1 & $1 / 6$ & 0.0953 & & \\
\hline media security D3 & 3 & 6 & 1 & 0.6548 & & \\
\hline
\end{tabular}

Table 9. The Comparison Matrix and the Check Results of First-level Evaluation Index, Information Security System E

\begin{tabular}{|c|c|c|c|c|c|c|}
\hline first-level evaluation indexes & \multicolumn{4}{|c|}{ information security system E } & \multirow[b]{2}{*}{$\begin{array}{c}\text { The } \\
\text { largest } \\
\text { eigenvalue }\end{array}$} & \multirow[b]{2}{*}{$\begin{array}{c}\text { The } \\
\text { consistency } \\
\text { ratio }\end{array}$} \\
\hline second-level evaluation indexes & \begin{tabular}{|c|} 
rules and \\
regulations \\
E1
\end{tabular} & $\begin{array}{c}\text { organization } \\
\text { E2 }\end{array}$ & $\begin{array}{l}\text { emergency } \\
\text { plan E3 }\end{array}$ & weight & & \\
\hline rules and regulations E1 & 1 & $1 / 3$ & $1 / 7$ & 0.0481 & \multirow{3}{*}{3.0324} & \multirow{3}{*}{0.0311} \\
\hline organization E2 & 3 & 1 & $1 / 4$ & 0.2109 & & \\
\hline emergency plan E3 & 7 & 4 & 1 & 0.7049 & & \\
\hline
\end{tabular}

Using the analytic hierarchy process (AHP) to calculate, the final results of computer network information security weight are shown in table 10. 
Table 10. The Evaluation Index Weight of Computer Network Information Security

\begin{tabular}{|c|c|c|c|c|}
\hline \multirow{20}{*}{$\begin{array}{l}\text { Computer network } \\
\text { information security } \\
\text { evaluation } \mathrm{P}\end{array}$} & $\begin{array}{l}\text { first-level evaluation } \\
\text { indexes }\end{array}$ & weight & $\begin{array}{l}\text { second-level evaluation } \\
\text { indexes }\end{array}$ & weight \\
\hline & \multirow{4}{*}{$\begin{array}{c}\text { network } \\
\text { communications } \\
\text { security A }\end{array}$} & \multirow{4}{*}{0.2162} & $\begin{array}{c}\text { communications line } \\
\text { security } A_{1}\end{array}$ & 0.1595 \\
\hline & & & $\begin{array}{c}\text { communications } \\
\text { control device security } \\
\mathrm{A}_{2}\end{array}$ & 0.6830 \\
\hline & & & $\begin{array}{c}\text { encryption measures A } \\
3 ;\end{array}$ & 0.0627 \\
\hline & & & access control $\mathrm{A}_{4}$ & 0.0949 \\
\hline & \multirow{5}{*}{$\begin{array}{l}\text { Information security } \\
\text { technology B }\end{array}$} & \multirow{5}{*}{0.0747} & $\begin{array}{l}\text { security auditing } \\
\text { function } \mathrm{B}_{1}\end{array}$ & 0.2217 \\
\hline & & & $\begin{array}{l}\text { system operation log } \\
\mathrm{B}_{2} ;\end{array}$ & 0.0389 \\
\hline & & & server backup $\mathrm{B}_{3}$ & 0.0662 \\
\hline & & & $\begin{array}{l}\text { anti-hacker attack } \\
\text { technology } \mathrm{B}_{4} ;\end{array}$ & 0.5619 \\
\hline & & & $\begin{array}{c}\text { antivirus technology } \\
\mathrm{B}_{5} ;\end{array}$ & 0.1112 \\
\hline & \multirow{4}{*}{ System security C } & \multirow{4}{*}{0.5586} & database security $\mathrm{C}_{1}$ & 0.0736 \\
\hline & & & $\begin{array}{l}\text { application software } \\
\text { security } \mathrm{C}_{2} ;\end{array}$ & 0.4709 \\
\hline & & & User authentication $\mathrm{C}_{3}$ & 0.1715 \\
\hline & & & $\begin{array}{c}\text { user information } \\
\text { backup } \mathrm{C}_{4}\end{array}$ & 0.2840 \\
\hline & \multirow{3}{*}{$\begin{array}{l}\text { Information physical } \\
\text { security D }\end{array}$} & \multirow{3}{*}{0.0435} & equipment security $\mathrm{D}_{1}$ & 0.2499 \\
\hline & & & $\begin{array}{c}\text { environment security } \\
\mathrm{D}_{2} ;\end{array}$ & 0.0953 \\
\hline & & & media security $\mathrm{D}_{3}$ & 0.6548 \\
\hline & \multirow{3}{*}{$\begin{array}{l}\text { Information security } \\
\text { system E }\end{array}$} & \multirow{3}{*}{0.1070} & $\begin{array}{l}\text { rules and regulations } \\
\qquad \mathrm{E}_{1} ;\end{array}$ & 0.0481 \\
\hline & & & organization $\mathrm{E}_{2}$ & 0.2109 \\
\hline & & & emergency plan $\mathrm{E}_{3}$ & 0.7049 \\
\hline
\end{tabular}

\subsection{Use the Fuzzy Comprehensive Evaluation Theory to Establish the Evaluation Model of Computer Network Information Security}

\subsubsection{The Introduction of Fuzzy Comprehensive Evaluation Theory}

Fuzzy comprehensive evaluation method is one of the most common methods used in fuzzy decision problems. Its aim is to use fuzzy mathematics knowledge system to determine the factor set and evaluation set of all evaluation indexes, structures the grade evaluation matrix and determines the evaluation levels of objects so as to make an overall evaluation to the objects. The basic process is shown as follows:

(1)Determine the evaluation index factor set $P=\left\{p_{1}, p_{2}, \cdots, p_{n}\right\}$, and there are $n$ evaluation indexes of the object. 
(2)Determine the level evaluation $\operatorname{set} V=\left\{v_{1}, v_{2}, \cdots, v_{m}\right\}$, and $m$ is the level number of the evaluation.

(3)Determine the fuzzy evaluation matrix $R=\left(r_{i j}\right)_{n \times m}$, the basic method is as follows:

First of all, make a level evaluation $f(p)(i=1,2, \cdots, n)$ to all factor $p$, we could get a fuzzy mapping $f$ from $P$ to $V$, namely

$$
f: P \rightarrow F(P), p_{i} \rightarrow f\left(p_{i}\right)=\left(r_{i 1}, r_{i 2}, \cdots, r_{i m}\right) \in F(V)
$$

Then, from the fuzzy mapping $f$, the fuzzy relation $R_{f} \in F(P \times V)$ could be induced, namely

$$
R_{f}\left(p_{i}, v_{i}\right)=f\left(p_{i}\right)\left(v_{i}\right)=r_{i j}, i=1,2, \cdots, n ; j=1,2, \cdots, m,
$$

The fuzzy evaluation matrix of the object could be determined as $R=\left(r_{i j}\right)_{n \times m}$.

(4)According to the evaluation index weight and the fuzzy evaluation matrix of the object, by using software MALAB, the comprehensive evaluation vector of the object $w=\gamma^{T} R$ could be calculated.

3.2.2 Construct the fuzzy comprehensive evaluation model of computer network information security.

(1)According to table 1 , the evaluation system of computer network information security, the factor set of computer network information security evaluation could be determined as $P=\left\{p_{1}, p_{2}, \cdots, p_{19}\right\}$, there are 19 evaluation indexes of computer network information security.

(2)Determine the level evaluation set of computer network information security $V=\left\{v_{1}, v_{2}, \cdots, v_{5}\right\}$. The evaluation results of computer network information security could be divided into five levels, that is, very safe, safe, general, dangerous, very dangerous. The specific classification of its factor set is shown in table 11 .

\section{Table 11. The Classification of the Evaluation Factor Set of Computer} Network Information Security

\begin{tabular}{|c|c|c|c|c|c|}
\hline factor set & \multicolumn{5}{|c|}{ level } \\
\hline $\begin{array}{c}\text { communications } \\
\text { line security A1; }\end{array}$ & very safe & safe & general & poor & very poor \\
\hline $\begin{array}{c}\text { communications } \\
\text { control device } \\
\text { security A 2; }\end{array}$ & very safe & safe & general & poor & very poor \\
\hline $\begin{array}{c}\text { encryption } \\
\text { measures A 3; }\end{array}$ & excellent & good & general & poor & very poor \\
\hline $\begin{array}{c}\text { access control A } \\
\text { 4; }\end{array}$ & very safe & safe & general & poor & very poor \\
\hline $\begin{array}{c}\text { security auditing } \\
\text { function B1; }\end{array}$ & excellent & good & general & poor & very poor \\
\hline $\begin{array}{c}\text { system operation } \\
\text { log B2; }\end{array}$ & excellent & good & general & poor & very poor \\
\hline $\begin{array}{c}\text { server backup B3; } \\
\text { anti-hacker attack } \\
\text { technology B4; }\end{array}$ & excellent & good & general & poor & very poor \\
\hline
\end{tabular}




\begin{tabular}{|c|c|c|c|c|c|}
\hline $\begin{array}{c}\text { antivirus } \\
\text { technology B5; }\end{array}$ & very high & high & general & low & very low \\
\hline $\begin{array}{c}\text { database security } \\
\text { C 1; }\end{array}$ & very safe & safe & general & poor & very poor \\
\hline $\begin{array}{c}\text { application } \\
\text { software security } \\
\text { C 2; }\end{array}$ & very safe & safe & general & poor & very poor \\
\hline $\begin{array}{c}\text { User } \\
\text { authentication C } \\
3 ;\end{array}$ & excellent & good & general & poor & very poor \\
\hline $\begin{array}{c}\text { user information } \\
\text { backup C4; }\end{array}$ & excellent & good & general & poor & very poor \\
\hline $\begin{array}{c}\text { equipment } \\
\text { security D1; }\end{array}$ & very safe & safe & general & poor & very poor \\
\hline $\begin{array}{c}\text { environment } \\
\text { security D2; }\end{array}$ & excellent & good & general & poor & very poor \\
\hline $\begin{array}{c}\text { media security } \\
\text { D3; }\end{array}$ & very safe & safe & general & poor & very poor \\
\hline $\begin{array}{c}\text { rules and } \\
\text { regulations E1; }\end{array}$ & very comprehensive & comprehensive & general & a few & few \\
\hline organization E2; & excellent & good & general & poor & very poor \\
\hline $\begin{array}{c}\text { emergency plan } \\
\text { E3; }\end{array}$ & excellent & good & general & poor & very poor \\
\hline
\end{tabular}

(3)Determine the fuzzy evaluation matrix of computer network information security evaluation $R=\left(r_{i j}\right)_{n \times m}$

With the help of expert evaluation method, carry on the level evaluation to the secondlevel evaluation indexes of computer network information security. The expert group is usually composed by 10 or more than 10 experts. Therefore, we could get:

$$
R_{A}=\left[\begin{array}{cccc}
r_{11}{ }^{(A)} & r_{12}{ }^{(A)} & \cdots & r_{15}{ }^{(A)} \\
\cdots & & \cdots & \cdots \\
r_{41}{ }^{(A)} & r_{42}{ }^{(A)} & \cdots & r_{45}{ }^{(A)}
\end{array}\right]
$$

$r_{i j}=$ the expert number of level $\mathrm{j} /$ the total number of experts

In the same way, we could get : $R_{B}, R_{C}, R_{D}, R_{E}$.

(4)According to the corresponding second-level index weight of computer network information security, the fuzzy evaluation matrix of 5 first-level evaluation indexes could be calculated as follows:

$$
R_{P}=\left(\begin{array}{lllll}
\gamma_{A}^{T} R_{A} & \gamma_{B}^{T} R_{B} & \gamma_{C}^{T} R_{C} & \gamma_{D}^{T} R_{D} & \left.\gamma_{E}^{T} R_{E}\right)^{T},
\end{array}\right.
$$

According to the weights of first-level evaluation indexes, the level evaluation vector of computer network information security could be obtained as follows:

$$
w=\gamma_{P}^{T} R_{P},
$$

Based on the maximum principle, the largest component of $w$ is the evaluation level of computer network information security. 


\section{An Empirical Analysis of Computer Network Information Security Evaluation}

Ten computer network experts give level evaluation to the computer network information security of Weifang University of Science and Technology. The evaluation results of 19 second-level evaluation indexes are shown in table 12:

Table 12. The Level Evaluation Results of the Computer Network Information Security of Weifang University of Science and Technology

\begin{tabular}{|c|c|c|c|c|c|}
\hline Factor set & \multicolumn{5}{|c|}{ level } \\
\hline communications line security A1; & 0 & 2 & 6 & 2 & 0 \\
\hline communications control device security A 2; & 1 & 2 & 4 & 2 & 1 \\
\hline encryption measures A 3; & 1 & 3 & 3 & 2 & 1 \\
\hline access control A 4; & 0 & 2 & 4 & 3 & 1 \\
\hline security auditing function B1; & 0 & 2 & 5 & 2 & 1 \\
\hline system operation log B2; & 0 & 1 & 7 & 1 & 1 \\
\hline server backup B3; & 2 & 4 & 4 & 0 & 0 \\
\hline anti-hacker attack technology B4; & 5 & 3 & 2 & 0 & 0 \\
\hline antivirus technology B5; & 3 & 5 & 1 & 1 & 0 \\
\hline database security C 1; & 1 & 3 & 5 & 1 & 0 \\
\hline application software security C 2; & 4 & 3 & 3 & 0 & 0 \\
\hline User authentication C 3; & 1 & 2 & 4 & 3 & 0 \\
\hline user information backup C4; & 0 & 2 & 6 & 1 & 1 \\
\hline equipment security D1; & 4 & 5 & 1 & 0 & 0 \\
\hline environment security D2; & 2 & 4 & 3 & 1 & 0 \\
\hline media security D3; & 1 & 3 & 5 & 1 & 0 \\
\hline rules and regulations E1; & 2 & 3 & 4 & 1 & 0 \\
\hline organization E2; & 1 & 3 & 6 & 0 & 0 \\
\hline emergency plan E3; & 3 & 2 & 4 & 1 & 0 \\
\hline
\end{tabular}

Carry on the data processing to the expert evaluation results of the computer network information security of Weifang University of Science and Technology. The details are shown in table 13. 


\section{Table 13. The Processed Data of Second-level Evaluation Index Level Evaluation Results of the Computer Network Information Security of Weifang University of Science and Technology}

\begin{tabular}{|c|c|c|c|c|c|}
\hline Factor set & \multicolumn{5}{|c|}{ level } \\
\hline communications line security A1; & 0 & 0.2 & 0.6 & 0.2 & 0 \\
\hline communications control device security A 2; & 0.1 & 0.2 & 0.4 & 0.2 & 0.1 \\
\hline encryption measures A 3; & 0.1 & 0.3 & 0.3 & 0.2 & 0.1 \\
\hline access control A 4; & 0 & 0.2 & 0.4 & 0.3 & 0.1 \\
\hline security auditing function B1; & 0 & 0.2 & 0.5 & 0.2 & 0.1 \\
\hline system operation log B2; & 0 & 0.1 & 0.7 & 0.1 & 0.1 \\
\hline server backup B3; & 0.2 & 0.4 & 0.4 & 0 & 0 \\
\hline anti-hacker attack technology B4; & 0.5 & 0.3 & 0.2 & 0 & 0 \\
\hline antivirus technology B5; & 0.3 & 0.5 & 0.1 & 0.1 & 0 \\
\hline database security C 1; & 0.1 & 0.3 & 0.5 & 0.1 & 0 \\
\hline application software security C 2; & 0.4 & 0.3 & 0.3 & 0 & 0 \\
\hline User authentication C 3; & 0.1 & 0.2 & 0.4 & 0.3 & 0 \\
\hline user information backup C4; & 0 & 0.2 & 0.6 & 0.1 & 0.1 \\
\hline equipment security D1; & 0.4 & 0.5 & 0.1 & 0 & 0 \\
\hline environment security D2; & 0.2 & 0.4 & 0.3 & 0.1 & 0 \\
\hline media security D3; & 0.1 & 0.3 & 0.5 & 0.1 & 0 \\
\hline rules and regulations E1; & 0.2 & 0.3 & 0.4 & 0.1 & 0 \\
\hline organization E2; & 0.1 & 0.3 & 0.6 & 0 & 0 \\
\hline emergency plan E3; & 0.3 & 0.2 & 0.4 & 0.1 & 0 \\
\hline
\end{tabular}

Using the program MATLB (The details are shown in the attachment.) to calculate, the evaluation vector of the computer network information security of Weifang University of Science and Technology could be obtained as follows:

$$
w=(0.1934,0.2481,0.4089,0.1098,0.0360),
$$

Based on the maximum principle we could get that the evaluation level of the computer network information security of Weifang University of Science and Technology is general.

\section{Conclusion}

Using fuzzy analytic hierarchy process to evaluate the computer network information security of Weifang University of Science and Technology, the result is accord with the actual situation, and the evaluation results are fair, reasonable and scientific. Computer network information security has become a focus of common concern of the government and our society. It is an important task for us to study the problems of computer network 
information security such as security policies, security technology, security management, security industry, information security infrastructure and the effective evaluation of information security. In this paper, the author studies the evaluation of computer network information security, gives a deep scientific understanding of computer network information security and the concepts, models, methods, tools and process of information security evaluation, which will help the correct solution to the network information security evaluation.

\section{Program MATLB}

$$
\begin{aligned}
& a=l o a d \text { ('wfkjxyjsjwlxxaq.txt'); } \\
& \mathrm{w}=[0.2162,0.0747,0.5586,0.0435,0.1070] \text {; } \\
& \mathrm{w} 1=[0.1595,0.6830,0.0627,0.0949] \text {; } \\
& \mathrm{w} 2=[0.2217,0.0389,0.0662,0.5619,0.1112] \text {; } \\
& \mathrm{w} 3=[0.0736,0.4709,0.1715,0.2840] \text {; } \\
& \text { w4 }=[0.2499,0.0953,0.6548] \text {; } \\
& \mathrm{w} 5=[0.0481,0.2109,0.7049] \text {; } \\
& \mathrm{b}(1,:)=\mathrm{w} 1 * \mathrm{a}([1: 4],:) \text {; } \\
& \mathrm{b}(2,:)=w 2 * a([5: 9],:) \text {; } \\
& \text { b }(3,:)=w 3 * a([10: 13],:) \text {; } \\
& \text { b }(4,:)=w 4 * a([14: 16],:) \text {; } \\
& \text { b }(5,:)=w 5 * a([17: \text { end }],:) \text {; } \\
& \mathrm{c}=\mathrm{w}^{*} \mathrm{~b} \\
& \mathrm{c}= \\
& \begin{array}{lllll}
0.1934 & 0.2481 & 0.4089 & 0.1098 & 0.0360
\end{array}
\end{aligned}
$$

\section{References}

[1] LI Zezhong, Ge Wenli, Deng Pu, Wang Xianpei,Analytic hierarchy process for reliability of computer networks[J],Engineering Journal of Wuhan University, 2008. 12(6): 113-117.

[2] Chuan-sheng xie, Xu Xin Hou Wentian, Xu Fengru, path analysis and synthetic evaluation of city low carbon economy development [J], technical economy, 2010 (8) : 29-32.

[3] Han Zhonggeng. Mathematical modeling method and its application [M]. Beijing: higher education press, 2005.

[4] Li Tao. Application of fuzzy mathematics in the supermarket customer degree of satisfaction evaluation [J]. Journal of capital normal university, 2015 (3): 15-18.

[5] Li Tao. Based on the network security risk fuzzy mathematical evaluation [J]. Journal of huaiyin normal university, 2015 (2): 60-64.

[6] Li Tao. The environment impact evaluation -based on urban land planning project [J]. International Journal of Earth Sciences and Engineering, 2015 (2).

[7] SiShouKui Sun Zhaoliang, mathematical modeling algorithm and its application [M]. Beijing: national defence industry press, 2015.

[8] Jing Peng. Information economics. Beijing: tsinghua university press. Version 1 August 2004.

[9] United States General Accounting Office, Accounting and Information Management Division Information Security Risk Assessment. August, 2002.

[10] National Computer Security Center. Department of Defense Trusted Computer System Evaluation Criteria 5200.28-STD, 2002.

[11] Davis, Beth. How secure is security. Computers\&Security.2001.

[12] Gordon, Sarah, Ford, Richard. Real world anti-virus product reviews and evaluations-Part 1. Network Seeurity. 2002 
[13] Iheagwara Charles, Blyth Andrew. Evaluation of the performance of ID systems in switched and distributed environment:the Real Secure case study. Computer Network.2002.

[14] Golic J D. Linear models for keystream generators. Computers, IEEE Transactions on, 2003.

[15] Schneier B.Cryptographic design vulner abilities. Computer, 2003.

[16] Joshi J, Ghafoor A, Aref W Getal. Digital government security in structure design challenges. Computer, 2001.

[17] Gordon Sarah, Ford Richard. Real world anti-virus product reviews and Evaluations-Part 2. Network Security.2003.

[18] Buzzard K. Computer security-what should you spend your money on. Computers\&Security.2002.

[20] BS7799-2:2002, Information Security Management-Part2: Specification for Information Security Management Systems[S], BSI 2002.

[21] Gerard E. Dallal. Introduction to Regression Models. http://www.tufts.edu /modell.html.

[22] Modiri N. The ISO reference model entities. IEEE Network, 1999.

[23] Gray Stoneburner, Alice Goguen, and Alexis Feringa. Risk Management Guide for Information Technology Security,NIST Special Publication,2003.

[24] I TSEC(The Information Technology Security Evaluation Criteria version 1.2)1999. 